\title{
Ceratium furcoides (Dinophyceae): UN DINOFLAGELADO INVASOR EN UN EMBALSE ALTO ANDINO ECUATORIAL EN COLOMBIA
}

\section{Ceratium furcoides (Dinophyceae): AN INVASIVE DINOFLAGELLATE IN AN EQUATORIAL HIGH ANDEAN RESERVOIR IN COLOMBIA}

\author{
Juan Carlos Jaramillo-Londoño
}

${ }^{1}$ Biólogo, Dr. en Biología, Grupo de Investigaciones y Mediciones Ambientales, Facultad de Ingenierías. Universidad de Medellín, Apartado Aéreo 1983, Fax (57-4)3405216. Medellín. Colombia; mail: jcjaramillo@udem.edu.co

\author{
Rev. U.D.C.A Act. \& Div. Cient. 21(1): 265-269, Enero-Junio, 2018 \\ https://doi.org/10.31910/rudca.v21.n1.2018.686
}

\section{INTRODUCCIÓN}

Los dinoflagelados del género Ceratium Schrank son habitantes frecuentes en muchos cuerpos de agua de regiones templadas de países, como Gran Bretaña, Canadá, Alemania, Hungría, Japón, Rumania, España, Suecia y Estados Unidos (Nicholls et al. 1980; Hickel, 1985; Lindström, 1992; Wu \& Chou, 1998; Caraus, 2012), en regiones subtropicales de Sudáfrica y de Australia (Whittington et al. 2000) y desde 1997, se han encontrado altas densidades en cuerpos de agua tropicales y subtropicales de Sudamérica, principalmente, en Argentina, en el sur de Brasil, en Chile y en Bolivia (Guerrero \& Echenique, 1997; Mac Donagh et al. 2005; Santos-Wisniewski et al. 2007; Matsumura-Tundisi et al. 2010; Oliveira et al. 2011; Boltovskoy et al. 2013; Cavalcante et al. 2013; 2016; Moreira et al. 2015; Almanza et al. 2016; Mariñelarena et al. 2016; Morales, 2016).

C. hirundinella y C. furcoides son especies similares, en cuanto a su contorno y tamaño y se pueden confundir entre sí. Una manera de distinguirlos es por la longitud de la placa 4'; mientras que en $C$. furcoides es muy corta y alcanza aproximadamente un tercio del cuerno apical, en C. hirundinella es mucho más larga y alcanza el ápice de la célula (Calado \& Larsen, 1997). En este estudio, los especímenes examinados tenían una placa 4' corta, que corresponde a C. furcoides (Figura 1).

En Colombia, llama la atención que C. furcoides ha sido encontrada, hasta el presente, en cuerpos de agua por encima de los $2.000 \mathrm{msnm}$; los primeros reportes fueron publicados por Ramírez et al. (2005), en el embalse de La Fe, ubicado a $2.155 \mathrm{~m} \mathrm{~s}$. n. m. Algunos años después, Bustamante et al. (2012) informaron de la presencia de C. furcoides en el embalse de Riogrande II, ubicado a $2.250 \mathrm{~m}$ s. n. m. En el presente trabajo, se reporta una floración de $C$. furcoides en otro embalse ecuatorial alto andino, el Embalse La García, ubicado en la región central del Departamento de Antioquia.

\section{MATERIALES Y MÉTODOS}

ElEmbalse La García, se encuentra a 20km al norte de Medellín y a 2.416 m s. n. m., en la región central del Departamento de Antioquia $\left(6^{\circ} 21^{\prime} 45^{\prime \prime} \mathrm{N}\right.$ y $75^{\circ} 36^{\prime} 21$ 'W). En esta zona, el clima es frío, la temperatura del aire oscila entre los 12 y los $16^{\circ} \mathrm{C}$. Se trata de un típico embalse alto andino ecuatorial, ubicado en una zona, con dos estaciones lluviosas muy marcadas, la primera de ellas, entre abril y junio y, la segunda, entre septiembre y noviembre, determinada, principalmente, por el desplazamiento de la Zona de Convergencia Intertropical (ZCIT). Las estaciones secas son de diciembre a marzo y de julio a agosto y la primera temporada es más seca que la segunda.

Las tres muestras fueron tomadas entre las 10:00 y las 15:00 horas, el 17 de junio, el 1 de agosto y 11 de septiembre de 2014 , respectivamente, en una única estación $\left(6^{\circ} 21^{\prime} 41.61 ” \mathrm{~N}\right.$ y $75^{\circ} 36^{\prime} 2.74$ 'W), de $11 \mathrm{~m}$ de profundidad, ubicada en cercanías al sitio de presa. La empresa Fabricato S.A. suministró los datos mensuales de precipitación. En cada visita, se utilizó un termómetro de mercurio, para medir la temperatura del aire y se midió la transparencia de la columna de agua, usando un disco 5 ecchi de $20 \mathrm{~cm}$ de diámetro, con bandas blancas y negras alternadas. A cada metro de profundidad, desde la superficie al fondo de la columna de agua, se realizaron perfiles verticales de temperatura del agua, oxígeno disuelto, conductividad eléctrica, sólidos 


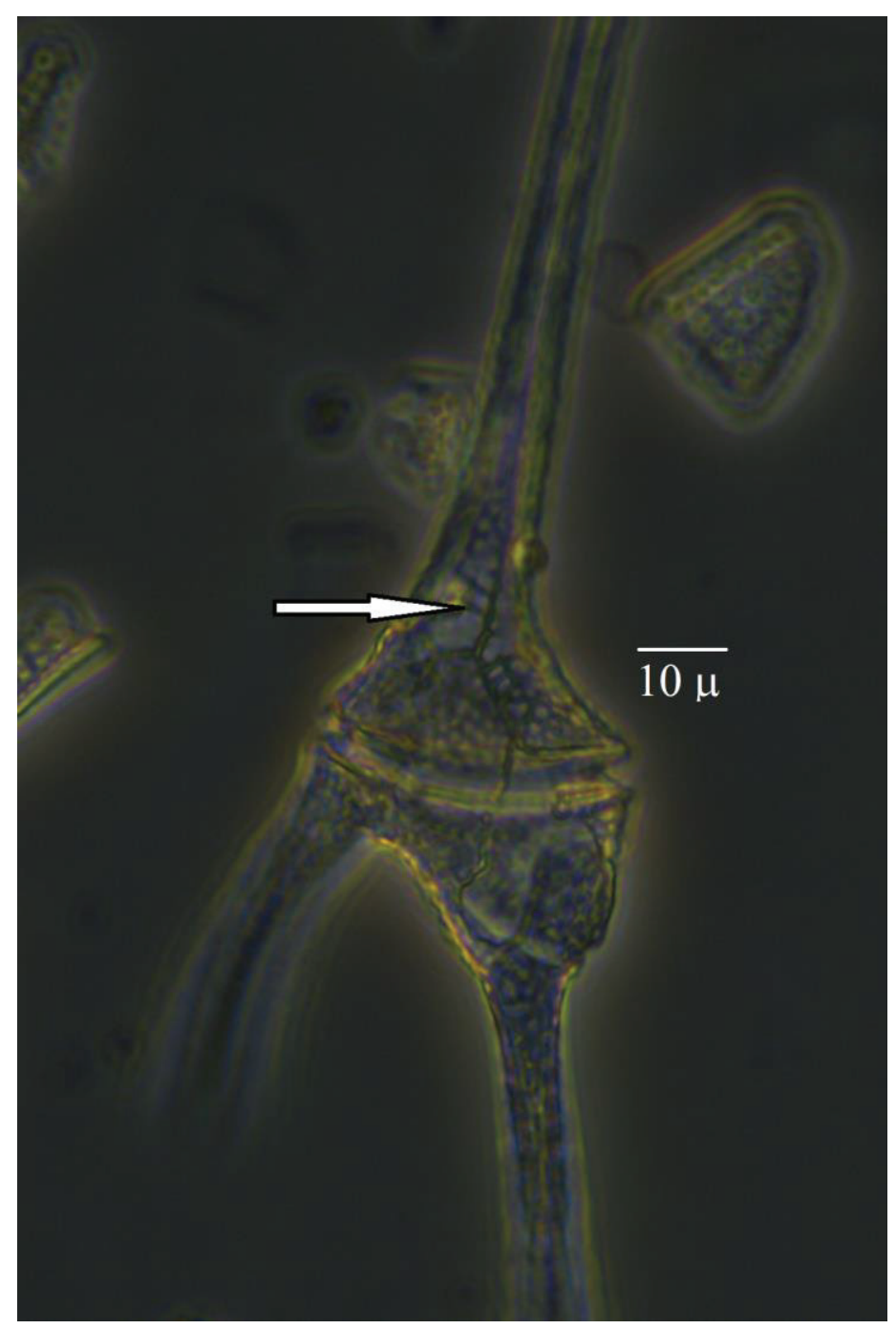

Figura 1. C. furcoides presente en el embalse la García, la flecha señala la placa 4'.

disueltos totales y $\mathrm{pH}$ usando un multiparámetro portátil Hach $^{\circledR}$ modelo HQ40D.

Las muestras de fitoplancton fueron tomadas a cinco profundidades (subsuperficie, mitad de la zona fótica, límite de la zona fótica, mitad de la columna de agua y el fondo), usando una botella muestreadora de $10 \mathrm{~L}$ de capacidad. Cada muestra recogida se filtró, a través de una red de plancton, con malla de $30 \mu \mathrm{m}$ y se preservó con una solución de lugol al 10\%; las muestras fueron transportadas al Laboratorio de Limnología de la Universidad de Medellín para su identificación y conteo. La densidad numérica, como ind/L de C. furcoides, se estimó contando alícuotas de $1 \mathrm{~mL}$ de las muestras fijas, en una celda de recuento SedgwickRafter, bajo un microscopio óptico Olympus ${ }^{\circledR}$ modelo CX23, con objetivo de 10X. El material recogido en este trabajo está depositado en el Laboratorio de Limnología de la Universidad de Medellín.

\section{RESULTADOS Y DISCUSIÓN}

Durante el período de estudio, los niveles mensuales de precipitación superaron los 140mm. La temperatura del aire, se mantuvo entre 19 y $23^{\circ} \mathrm{C}$. La zona eufótica no superó los $3 \mathrm{~m}$ de profundidad. La temperatura del agua fue inferior a $22^{\circ} \mathrm{C}$. La transparencia, la conductividad eléctrica y los sólidos totales disueltos fueron bajos, el pH era alcalino y el oxígeno disuelto en la superficie del embalse fue alto (Tabla 1). 
Tabla 1. Variables medidas en el embalse La García en una estación cercana al sitio de presa durante los tres muestreos realizados.

\begin{tabular}{|l|c|c|c|}
\hline \multicolumn{1}{|c|}{ Variable } & $\begin{array}{c}\text { Muestreo 1 } \\
\mathbf{( 1 7 . 0 6 . 2 0 1 4 )}\end{array}$ & $\begin{array}{c}\text { Muestreo 2 } \\
\mathbf{( 0 1 . 0 8 . 2 0 1 4 )}\end{array}$ & $\begin{array}{c}\text { Muestreo 3 } \\
\mathbf{( 1 1 . 0 9 . 2 0 1 4 )}\end{array}$ \\
\hline Precipitación mensual (mm) & 142 & 65 & 180 \\
\hline Temperatura del aire $\left({ }^{\circ} \mathrm{C}\right)$ & 23 & 20 & 19 \\
\hline Temperatura del agua $\left({ }^{\circ} \mathrm{C}\right)$ Sup & 22 & 19 & 16 \\
\hline Temperatura del agua $\left({ }^{\circ} \mathrm{C}\right)$ Fon & 19 & 17 & 0,8 \\
\hline Transparencia $(\mathrm{m})$ & 1,1 & 9,8 & 8,4 \\
\hline pH & 10,9 & 47,6 & 37,4 \\
\hline Conductividad eléctrica ( $\mu$ S/cm) & 50,9 & 11,9 & 5,0 \\
\hline Oxígeno disuelto $(\mathrm{mg} / \mathrm{L})$ Sup & 8,3 & 1,8 & 1.3 \\
\hline Oxígeno disuelto $(\mathrm{mg} / \mathrm{L})$ Fon & 3,3 & 18,8 & 21,9 \\
\hline Sólidos totales disueltos (mg/L) & 26,8 & 74839 & 111236 \\
\hline Densidad total C.furcoides (ind/L) & 22322 & 25864 & 6636 \\
\hline Densidad C.furcoides (ind/L) Sup & 2989 & 25040 & 52936 \\
\hline Densidad C.furcoides (ind/L) MZF & 16225 & 17503 & 40196 \\
\hline Densidad C.furcoides (ind/L) LZF & 2366 & 1320 & 4472 \\
\hline Densidad C.furcoides (ind/L) Fon & 10 & & \\
\hline
\end{tabular}

Sup: superficie; MZF: Mitad de la zona fótica; LZF: Límite de la zona fótica; Fon: Fondo.

La temperatura y el oxígeno disuelto en la columna de agua mostraron un perfil clinógrado, revelando una ligera estratificación térmica y una drástica caída de oxígeno disuelto en el fondo (Tabla 1).

En el embalse La García, la población de C. furcoides alcanzó una densidad total de 208397ind/L. Las densidades más altas, se encontraron hacia la mitad de la zona fótica (94201ind/L), donde los niveles de luz son menores y las densidades más bajas, hacia el fondo del embalse (5802ind/L).

Las densidades de $C$. furcoides halladas son mayores cuando se comparan con las densidades reportadas por Bustamante et al. (2012), en el embalse de Riogrande II (41213ind/L) y superior a las densidades registradas en otros embalses del continente sudamericano. En el embalse de Furnas, Silva et al. (2012) reportaron densidades de 28564ind/L y en el Yaciretá, Meichtry et al. (2014) informaron densidades de 15000ind/L; sin embargo, los valores encontrados en el embalse La García son inferiores comparados con los reportados por Matsumura-Tundisi et al. (2010), en el embalse Billings, donde encontraron densidades superiores a 535209ind/L. Estas altas densidades halladas han sido relacionadas con procesos de eutrofización en estos embalses; no obstante, se hace necesario realizar un estudio más detallado para establecer realmente cuál es el estado trófico del embalse La García y cuál es su relación con la población de C. furcoides presente.
Los individuos de $C$. furcoides pueden alterar su posición vertical en la columna de agua nadando, lo que les permite mantener una profundidad óptima en términos de luz, concentración de oxígeno y nutrientes (Whittington et al. 2000). C. furcoides evita entornos anóxicos y altas intensidades de luz superficial; es por ello, que se encontraron las densidades más altas de $C$. furcoides en el centro de la zona fótica (Tabla 1). Esta especie tiende a alejarse de las capas más profundas del embalse, donde las concentraciones de oxígeno disuelto fueron más bajas $(<3,3 \mathrm{mg} / \mathrm{L})$, así como en un conglomerado en capas de agua donde recibe, al menos, el $50 \%$ de la luz incidente en la superficie y donde las concentraciones de oxígeno disuelto son más altas (> 7,0 mg/L).

Claramente, los primeros reportes de la presencia de esta especie ocurrieron en regiones templadas en el Hemisferio Norte, después la especie alcanzó la región tropical y subtropical en el Hemisferio Sur, principalmente, en América del Sur y de ahí se expandió, gradualmente, hacia la Zona Andina Ecuatorial (Moreira et al. 2015; Cavalcante et al. 2016; Meichtry et al. 2016). La rápida dispersión y el establecimiento de esta especie pueden estar asociadas a rasgos considerados comunes a los organismos invasores, como son sus altas tasas de crecimiento, una alta eficiencia en la utilización de los recursos disponibles y una capacidad competitiva superior a la de las especies nativas, cuando las condiciones locales varían. Su gran tamaño evita la depredación por parte del zooplancton, la presencia de 
flagelos le permite realizar migraciones a estratos profundos ricos en nutrientes en diferentes momentos del día, así como cambiar su distribución con respecto a los gradientes de luz. Con la formación de quistes que se sedimentan pueden sobrevivir en condiciones ambientales extremas y mantenerse quiescentes durante largos períodos de tiempo, de esta manera, cumplen un importante papel ecológico, como fuente de inóculo de floraciones recurrentes de esta especie (Matzuoka \& Fukuyo, 2000). Todas estas características morfológicas y ecofisiológicas definen el éxito de la especie, para su permanencia en este embalse.

Un paso importante para poder entender la distribución de $C$. furcoides es estudiar su potencial de dispersión pasivo y los factores que pueden hacer un evento de dispersión efectivo, permitiendo la colonización de un nuevo ecosistema, en el que las condiciones limnológicas y climáticas son apropiadas para su crecimiento. Actualmente, no es posible establecer cómo llegó esta especie a Colombia; sin embargo, puede estar asociado a modificaciones del régimen hidrológico, a cambios en la calidad del agua o al transporte de sus estados de resistencia (quistes) por aves, por insectos acuáticos o por intervención antrópica. Por otro lado, se hace necesario realizar un trabajo mucho más detallado y prolongado, con el fin de establecer la dinámica de la población de este dinoflagelado y determinar cuál es el efecto que su población está ocasionando sobre la comunidad planctónica, en este cuerpo de agua.

Agradecimientos: El autor desea agradecer a Jorge Sanabria Agudelo por su colaboración, a la empresa Fabricato S.A., por el permiso otorgado para la realización de los muestreos y a los revisores anónimos, quienes con sus comentarios contribuyeron a mejorar el manuscrito. Conflicto de Intereses: El manuscrito fue preparado y revisado por el autor, quien declara que no existe conflicto de intereses, que ponga en riesgo la validez de los resultados presentados.

\section{BIBLIOGRAFÍA}

1. ALMANZA, V.; BICUDO, C.E.M.; PARRA, O.; URRUTIA, R. 2016. Características morfológicas y limnológicas de las floraciones de Ceratiunm furcoides (Dinophyta) en un lago somero de Chile Central. Limnética. 35(1):253-268.

2. BOLTOVSKOY, A.; ECHENIQUE, R.; GUERRERO, J.M. 2013. Sucesivas invasiones de especies de Ceratium (Dinophyceae) en Sudamérica: un proceso que lleva dos décadas. Bol. Soc. Argent. Bot. 48:27.

3. BUSTAMANTE, C.; RAMÍREZ, J.J.; BOLTOVSKOY, A.; VALLEJO, A. 2012. Spatial and temporal change characterization of Ceratium furcoides (Dinophyta) in the equatorial reservoir Riogrande II, Colombia. Acta Limnol. Bras. 24(2):207-219.

4. CALADO, A.J.; LARSEN, J. 1997. On the identity of the type species of the genus Ceratium Schrank (Dinophyceae), with notes on C. hirundinella. Phycologia. 36(6):500-505.

5. CARAUS, I. 2012. Algae of Romania. A distributional checklist of actual algae. Version 2.3. Third revision. Bacau: Univ. Bacau.

6. CAVALCANTE, K.P.; CARDOSO, L.S.; SUSSELLA, R.; BECKER, V. 2016. Towards a comprehension of Ceratium (Dinophyceae) invasion in Brazilian freshwaters: autecology of $C$. furcoides in subtropical reservoirs. Hydrobiologia. 771(1):265280.

7. CAVALCANTE, K.P.; ZANOTELLI, J.C.; MÜLLER, C.C.; SCHERER, K.D.; FRIZZO, J.K.; LUDWIG, T.A.V.; CARDOSO, L.S. 2013. First record of invasive Ceratium (Schrank, 1793) species (Dinophyceae) in Southern Brazil, with notes on their dispersive patterns in Brazilian environments. Check List. 9(4):862-866.

8. GUERRERO, J.; ECHENIQUE, R. 1997. Ceratium hirundinella blooms in Argentine reservoirs. Harmful Algae News.16:3.

9. HICKEL, B. 1985.The population structure of Ceratium in a small eutrophic lake. Verh. Internat. Verein. Limnol. 22:2845-2849.

10. LINDSTRÖM, K. 1992. Ceratium in Lake Erken: vertical distribution, migration and form variation. Nord. J. Bot. 12(5):541-556.

11. MAC DONAGH, M.E.; CASCO, M.A.; CLAPS, M.C. 2005. Colonization of a Neotropical Reservoir (Córdoba, Argentina) by Ceratium hirundinella (O. F. Müller) Bergh. Ann. Limnol-Int. J. Lim. 4(4):291-299.

12. MARIÑELARENA, A.; MAC DONAGH, M.E.; DONADELLI, J.; CASCO, M.A. 2016. Un caso inusual de eutrofización en el embalse río Tercero: El posible rol de dos bioinvasores. Biología Acuática. 31:10-18.

13. MATSUMURA-TUNDISI, T.; TUNDISI, J.G.; LUZIA, A.P.; DEGANI, R.M. 2010. Occurrence of Ceratium furcoides (Levander) Langhans 1925 bloom at the 
Billings Reservoir, São Paulo State, Brazil. Braz. J. Biol. 70(3):825-829.

14. MATZUOKA, K.; FUKUYO, Y. 2000. Technical guide for modern dinoflagellate cyst study. WESTPAC-HAB/ WESTPAC/IOC. 78p.

15. MEICHTRY, N.; BOLTOVSKOY, A.; ROJAS, C.; RODRÍGUEZ, R.M. 2014. Primer registro del dinoflagelado invasor Ceratium furcoides (Levander) Langhans 1925 en la Argentina y su distribución en el área de influencia del Embalse Yacyretá (río Paraná, Argentina-Paraguay). Limnetica. 33(1):153160.

16. MEICHTRY, N.M.; VOGLER, R.E.; MOLINA, M.J.; LLANO, V.M. 2016. Potential distribution of the invasive freshwater dinoflagellate Ceratium furcoides (Levander) Langhans (Dinophyta) in South America. J. Phycol. 52(29):200-208.

17. MORALES, E.A. 2016. Floración de Ceratium furcoides (Levander) Langhans (Dinoflagellata, Dinophyceae) en la represa de La Angostura, Cochabamba, Bolivia. Acta Nova. 7(4):389-398.

18. MOREIRA, R.A.; ROCHA, O.; SANTOS, R.M.; LAUDARESSILVA, R.; DIAS, E.S.; ESKINAZI-SANT'ANNA, E.M. 2015. First record of Ceratium furcoides (Dinophyta), an invasive species, in a temporary high-altitude lake in the Iron Quadrangle (MG, Southeast Brazil). Braz. J. Biol. 75(1):98-103.

19. NICHOLLS, K.M.; KENNEDY, W.; HANNET, C. 1980. A fish kill in Heart Lake, Ontario, associated with the collapse of a massive population of Ceratium hirundinella (Dinophyceae). Freshwater Biol. 10(6):553-561.
20. OLIVEIRA, H.S.B.; MOURA, A.N.; CORDEIRO-ARAÚJO, M.K. 2011. First record of Ceratium Schrank, 1973 (Dinophyceae: Ceratiaceae) in freshwater ecosystems in the semiarid region of Brazil. Check List. 7(5):626628.

21. RAMÍREZ, J.J.; GUTIÉRREZ, F.L.; VARGAS, A. 2005. Respuesta de la comunidad fitoplanctónica a experimentos de eutrofización artificial realizados en la represa La Fe, El Retiro, Antioquia, Colombia. Caldasia. 27(1):103-115.

22. SANTOS-WISNIEWSKI, M.J.; SILVA, L.C.; LEONE, I.C.; LAUDARES-SILVA, R.; ROCHA, O. 2007. First Record of the occurrence of Ceratium furcoides (Levander) Langhans 1925, an invasive species in the hydroelectricity power plant Furnas reservoir, MG, Brazil. Braz. J. Biol. 67(4):791-793.

23. SILVA, L.C.; LEONE, I.C.; SANTOS-WISNIEWSKI, M.J.; PERET, A.C.; ROCHA, O. 2012. Invasion of the dinoflagellate Ceratium furcoides (Levander) Langhans 1925 at tropical reservoir and its relation to environmental variables. Biota Neotrop. 12(2):1-8.

24. WHITTINGTON, J.; SHERMAN, B.; GREEN, D.; OLIVER, R.L. 2000. Growth of Ceratium hirundinella in a subtropical Australian reservoir: the role of vertical migration. J. Plankton Res. 22(6):1025-1045.

25. WU, J.T.; CHOU, J.W. 1998. Dinoflagellate associations in Feitsui Reservoir. Bot. Bull. Acad. Sin. 39(2):137145.

Recibido: Diciembre 12 de 2017

Aceptado: Marzo 24 de 2018

Cómo citar:

Jaramillo-Londoño, J.C. 2018. Ceratium furcoides (Dinophyceae): un dinoflagelado invasor en un embalse alto andino ecuatorial en Colombia. Rev. U.D.C.A Act. \& Div. Cient. 21(1): 265-269. 